\title{
Laser Ablated Silver Nanoparticles with Nearly the Same Size in Different Carrier Media
}

\author{
Antonio M. Brito-Silva, ${ }^{1,2}$ Luiz A. Gómez, ${ }^{3}$ Cid B. de Araújo, ${ }^{3}$ and André Galembeck ${ }^{4,2}$ \\ ${ }^{1}$ Programa de Pós-graduação em Ciência de Materiais, CCEN, UFPE, 50670-901 Recife, PE, Brazil \\ ${ }^{2}$ Centro de Tecnologias Estratégicas do Nordeste, Av. Prof. Luiz Freire, 01, 50670-90 Recife, PE, Brazil \\ ${ }^{3}$ Departamento de Física, UFPE, 50670-901 Recife, PE, Brazil \\ ${ }^{4}$ Departamento de Química Fundamental, UFPE, 50740-540 Recife, PE, Brazil \\ Correspondence should be addressed to André Galembeck, andre@ufpe.br
}

Received 16 August 2009; Revised 20 February 2010; Accepted 22 March 2010

Academic Editor: Jun Li

Copyright (C) 2010 Antonio M. Brito-Silva et al. This is an open access article distributed under the Creative Commons Attribution License, which permits unrestricted use, distribution, and reproduction in any medium, provided the original work is properly cited.

\begin{abstract}
Poly(vinyl-pyrrolidone) (PVP) stabilized silver nanoparticles with an average particle size ranging from 4.3 to $4.9 \mathrm{~nm}$ were synthesized by laser ablation in preformed colloids in methanol, acetone, ethylene glycol, and glycerin. Aqueous colloids obtained using PVP, poly(vinyl-alcohol) (PVA), and sodium citrate as stabilizing agents also lead to a good control over particle size distribution. Silver ions were reduced with sodium borohydride. The smaller average particle size and narrower dispersivity in comparison to previously reported data were ascribed to the relatively small size of the particles formed in the chemical reduction step, laser fluence, and the use of PVP, which was not previously used as the stabilizing agent in "top-down" routes. The surface plasmon resonance band maximum wavelength shifted from $398 \mathrm{~nm}$ in methanol to $425 \mathrm{~nm}$ in glycerin. This shift must be due to solvent effects since all other variables were the same.
\end{abstract}

\section{Introduction}

Silver nanoparticles (Ag NPs) have been extensively studied due to their many potential applications [1-9]. While a narrow particle size distribution (PSD) is not required when exploring their action as antimicrobial agents [8] or in solar control glazing [9], it is very important when considering their optical [10] and electronic properties [2,3].

In order to investigate the effect of the surrounding medium on the optical properties of metallic colloids, it is essential to develop methodologies to prepare colloids in which NPs with nearly the same size can be obtained in different liquids and stabilizing agents.

The choice of the metal precursor, the liquid phase, and the reducing and protective agents as well as the experimental parameters, such as metal-to-reducing (and metalto-stabilizing) agent stoichiometric ratios, order of reagent addition, and temperature, influence the size and shape of the resulting nanoparticles [11-19]. Small changes in the experimental parameters can lead to dramatic variations in the resulting samples $[11,17]$.
Bottom-up approaches have proven to be more effective if one is interested in silver colloids with a narrow PSD. Good results were obtained by chemical reduction at room temperature [11] or while heating [12-15], photoreduction $[16]$, and microwave $[17,18]$ or gamma irradiation [19, 20]. However, if the liquid phase is changed while all other variables are fixed, then different average particle sizes will result, changing the size-dependent properties of the colloid [17]. Also, nanoparticles with the same size that are prepared from different reagents and synthesized with different methodologies in different liquids will not be directly comparable in optical experiments.

Samples resulting from laser ablation, a typical top-down route, can be produced in arbitrary solutions, and in this method, several experimental variables are strictly related to the laser operating conditions, such as the photon energy of the laser light and the pulse number [21-25]. Ag colloids in acetone, water, and ethanol with 5, 13, and $22 \mathrm{~nm}$ average particle sizes, respectively, were prepared by laser ablation by Tilaki et al. without using stabilizing agents, and the resulting samples presented a broad PSD [21]. 


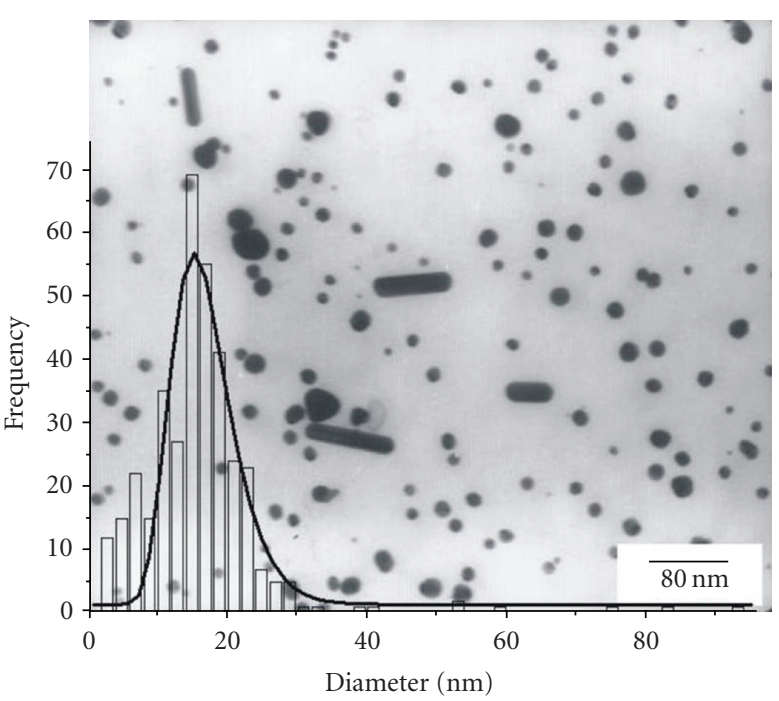

(a)

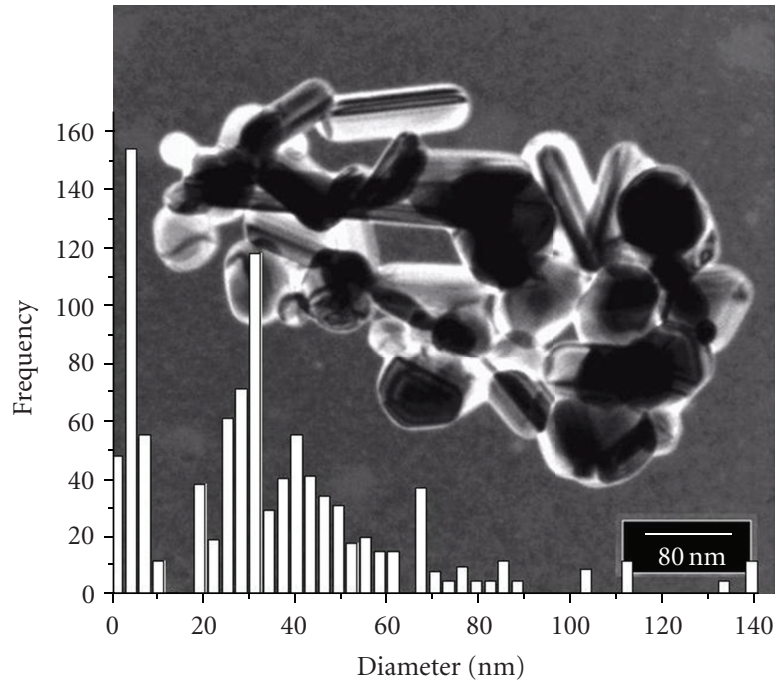

(b)

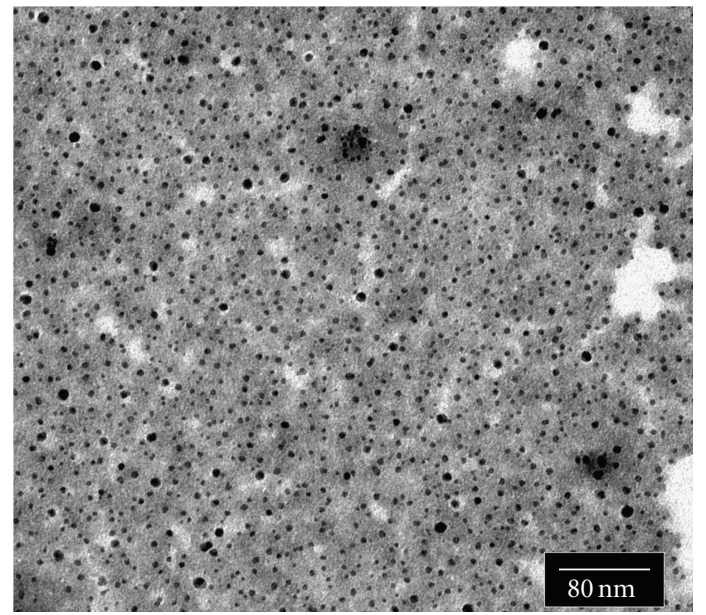

(c)

Figure 1: Transmission electron microscopy and particle size distribution of silver nanoparticles formed by chemical reduction of $\mathrm{Ag}^{+}$with sodium borohydride. (a) In methanol/PVP; (b) and (c) in water/sodium citrate.

Our research group has been investigating the optical properties of Ag NPs in both liquid and solid media [26-28]. In order to investigate the effect of the surrounding medium on the nonlinear optical properties of silver colloids, a new route to synthesize silver nanoparticles of the same size in different liquid media and with different stabilizing agents in the same liquid was developed and is described in this paper.

\section{Methodology}

Silver colloids were prepared in methanol, acetone, ethyleneglycol, glycerin, and deionized water using poly(vinyl pyrrolidone) (PVP, from Aldrich) as the stabilizing agent. In water, poly(vinyl-alcohol) (PVA) and sodium citrate (Aldrich) were also employed. A $4.71 \times 10^{-2} \mathrm{~mol} \cdot \mathrm{L}^{-1}$ silver nitrate (Vetec) methanolic solution was used as the source of silver ions in all experiments.
In a typical procedure, PVP $\left(0.25 \mathrm{~g}, 40,000 \mathrm{~g} \cdot \mathrm{mol}^{-1}\right)$ and sodium borohydride $\left(2.91 \times 10^{-4} \mathrm{~mol}\right)$ were dissolved in $250 \mathrm{~mL}$ of the liquid phase. The silver solution $(1.4 \mathrm{~mL})$ was subsequently added with strong stirring, and the sample heated up to the boiling point at reflux, which was kept for 1 hour. The same procedure applies to samples prepared using sodium citrate and PVA as stabilizing agents.

A 1-mL aliquot of each sample was transferred to a cuvette and irradiated while stirring with a pulsed (8ns) $\mathrm{Nd}$ :YAG laser at $532 \mathrm{~nm}$ (2nd harmonic) with a $10 \mathrm{~Hz}$ repetition rate. Laser power and fluence were $85 \mathrm{~mJ} /$ pulse and $221 \mathrm{~mJ} \cdot \mathrm{cm}^{-2}$, respectively. The samples were irradiated for 30 minutes, leading to the formation of transparent yellow colloidal suspensions. The colloids prepared with PVA were stable for 8 days; when PVP and sodium citrate were used, the samples were stable for over than 5 months.

The synthetic route described above was developed on the basis of the reports by Kamat [29], Bell [30], and Šmejkal 


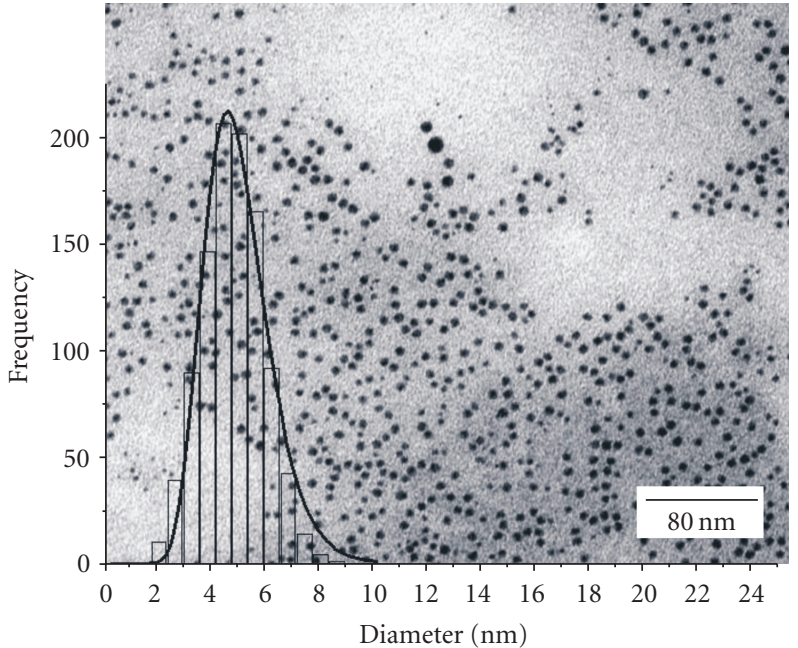

(a)

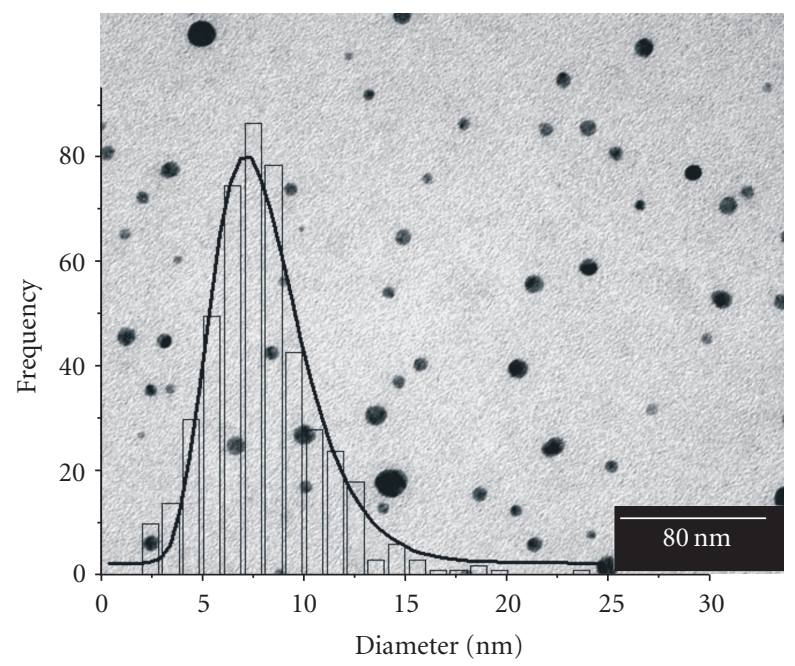

(c)

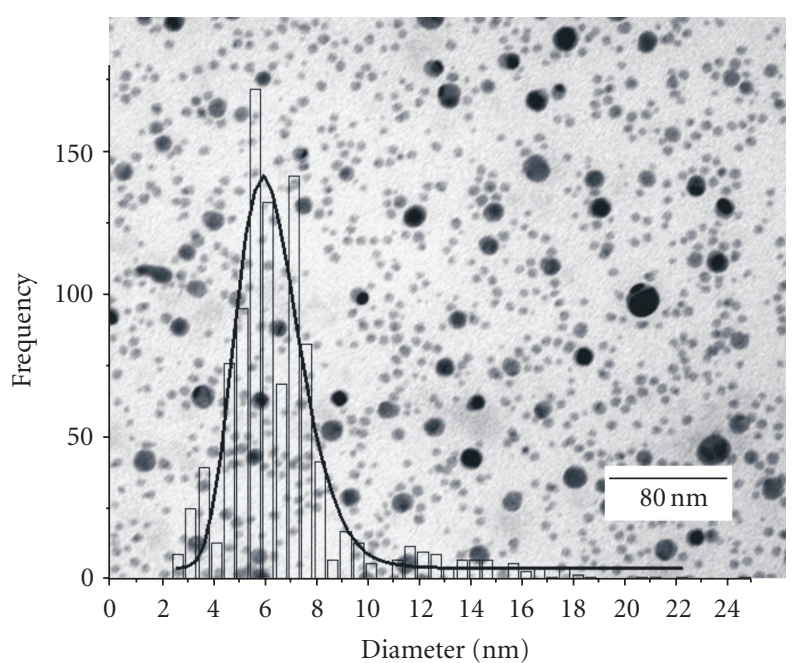

(b)

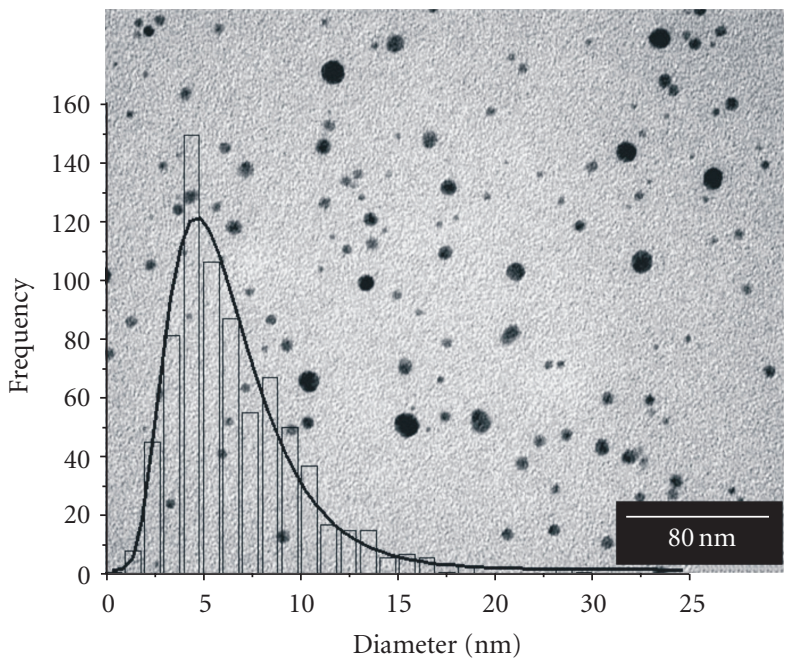

(d)

FIGURE 2: Transmission electron microscopy images and particle size distribution of silver nanoparticles after laser ablation. The samples are referred as "liquid/stabilizing agents." (a) Methanol/PVP; (b) water/PVP; (c) acetone/PVP; (d) water/sodium citrate.

[31]. However, the use of PVP as a stabilizing agent in laser-ablated silver colloids was not reported earlier, and silver solution addition to a liquid that already contains the reducing agent is not the usual procedure.

The samples were characterized by transmission electron microscopy with a JEOL $80 \mathrm{kV}$ instrument. One drop of a diluted silver colloidal suspension was deposited on a carbon-copper grid and allowed to dry at room temperature. The mean particle sizes, standard deviations, and histograms were calculated by counting 400-1000 particles for each sample. UV-visible absorption spectra were acquired in the 190-900 nm range, in transmission mode.

\section{Results and Discussion}

The preparation of silver colloids by laser ablation can be achieved directly from silver targets [21-24,32] or preformed colloids $[25,29,30]$. The latter approach was carried out in this study.

Transmission electron microscopy (TEM) images from silver nanoparticles resulting from the chemical reduction step in methanol/PVP and water/sodium citrate and their respective particle size distribution histograms are presented in Figure 1. PVP-stabilized samples present smaller and mainly spherical nanoparticles, as presented in Figure 1(a). Elliptical particles and rods with a large aspect ratio also appear. The histogram was plotted measuring the largest axis for the nonsymmetrical particles, which gave a $16.4 \pm 9.3 \mathrm{~nm}$ average particle size.

A much broader PSD $(34 \pm 27.4 \mathrm{~nm})$ was observed when sodium citrate was used as the capping agent. While many small spherical nanoparticles appeared (Figure 2(c)), bigger particles with sharp edges and rods were also found (Figure 2(b)). 


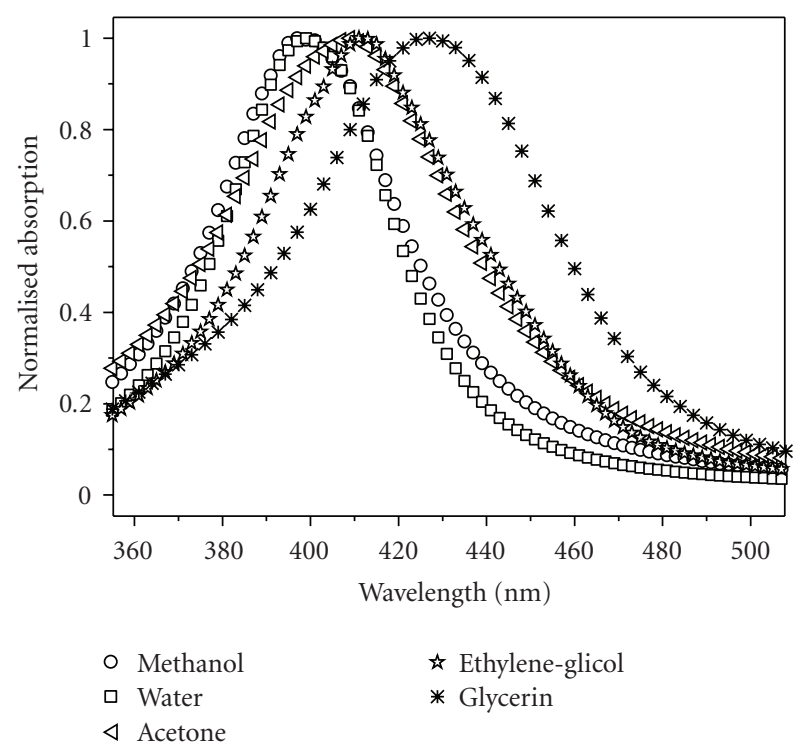

Figure 3: Absorption spectra of PVP protected silver nanoparticles in different liquids: (০) methanol; ( $\square$ ) water; ( $\triangleleft)$ acetone; ( $\nleftarrow$ ) ethylene glycol; (*) glycerin.

TABle 1: Average particle size and standard deviations for laserablated silver nanoparticles in methanol, water, ethylene glycol, acetone, and glycerin, using PVP, PVA, and sodium citrate as stabilizing agents. L/SA stands for liquid/stabilizing agent.

\begin{tabular}{lccc}
\hline L/SA & Size \pm SD/nm & L/SA & Size \pm SD/nm \\
\hline methanol/PVP & $4.9 \pm 1.2$ & water/PVP & $6.3 \pm 2.7$ \\
acetone/PVP & $4.9 \pm 2.0$ & water/PVA & $7.8 \pm 2.7$ \\
ethylene-glicol/PVP & $4.3 \pm 2.5$ & water/citrate & $5.8 \pm 3.3$ \\
glycerin/PVP & $4.4 \pm 2.4$ & & \\
\hline
\end{tabular}

When the samples formed by chemical reduction were submitted to laser ablation, smaller spherical NPs with a narrower PSD resulted. The results are summarized in Table 1. Figure 2 shows TEM images and histograms from samples prepared in methanol, water, and acetone using PVP as the stabilizing agent and from citrate-protected nanoparticles in water.

When PVP-protected NPs in organic solvents are considered, the average particle size is within the 4.3 to $4.9 \mathrm{~nm}$ range irrespective of the liquid medium, as presented in the first column of Table 1. A very high degree of particle size control was achieved as compared to other reported silver colloids dealing with silver nanoparticle preparation in different carrier media [17, 21, 23, 32].

The sample prepared in water/PVP presented bigger particles with a broader dispersion and showed a bimodal PSD with particles in the $10-18 \mathrm{~nm}$ range (Figure 2(b)). The other samples synthesized in water also presented bigger NPs than the samples synthesized in organic media. If the samples are compared before and after laser irradiation (see Figures 1(a) and 2(a) for methanol and Figures 1(b) and 2(d) for water), one can see that the particles that were formed in methanol already present an average diameter smaller than $20 \mathrm{~nm}$ after chemical reduction. Hence, good control on the chemical reduction step contributes to obtaining better control after laser ablation. A better control over particle size in samples prepared using citrate can be achieved with more dilute samples [33]. However, for the purpose of this work, it was important to maintain the $\mathrm{Ag}^{+}$concentration and to get samples with nearly the same particle size starting from the same initial experimental conditions in all liquids.

Tilaki et al. reported that the particle size of silver NPs formed by laser ablation was dependent on the liquid medium for water, acetone, and ethanol. In their work, however, laser ablation was performed directly on silver foils, and no stabilizing agent was used [21]. Ganeev et al. prepared Ag-NPs ranging from 5 to $10 \mathrm{~nm}$ in ethylene glycol, water, and ethanol, but they did not provide detailed information about particle morphology [32].

Samples prepared directly from macroscopic silver targets using surfactants (sodium dodecyl sulfate and cethyltrimethylammonium bromide) or citric acid as protective agents lead to samples with bigger particles and with a broader particle size distribution [17, 22, 34].

A mechanism for particle size reduction based on melting and vaporization of the pristine nanoparticles as a result of the strong absorption of the laser energy by the particles and low heat transfer for the surrounding medium was proposed by Takami et al. for gold nanoparticles in water/citric acid [35]. More recently, Pyatenko et al. stated that the same mechanism also applies to silver nanoparticles and that there is minimum laser fluence needed for particle heating, melting, and vaporization by one laser pulse, which is a function of the particle diameter. Silver NPs with a size of $8.1 \pm 1.7 \mathrm{~nm}$ were obtained in a water/citric acid system when laser fluences $J>0.18 \mathrm{~J} \cdot \mathrm{cm}^{-2}$ were used [36].

The colloids described in this report were prepared with a $0.221 \mathrm{~J} \cdot \mathrm{cm}^{-2}$ fluence, which is above this threshold. Hence, the mechanism of NPs formation in this case must be the same as the one described by the authors cited above.

Although PVP is widely used as a stabilizing agent when silver nanoparticles are synthesized by chemical methods, it has not been explored in laser-ablated colloids; the most commonly used stabilizers are sodium citrate and surfactant molecules. According to Qiao et al., Ag NPs smaller than $50 \mathrm{~nm}$, which is the case in our study, are stabilized by the coordination of the nitrogen atoms in PVP to the metallic surface [37]. Also, PVP forms coordinative complexes with silver ions, which are more easily reduced than silver ions in aqueous solution [38]. An X-ray photoelectron spectroscopy carried out by Huang et al., however, claims that the polymer interacts with silver particles through the oxygen atom in the $>\mathrm{C}=\mathrm{O}$ group [39].

Hence, we ascribe the smaller average particle size and the particle size distribution of our samples prepared in organic solvents, in comparison to other reports in the literature, to three factors: the relatively small size of particles obtained in the chemical reduction step, laser fluence, and the role of PVP, as described above. 


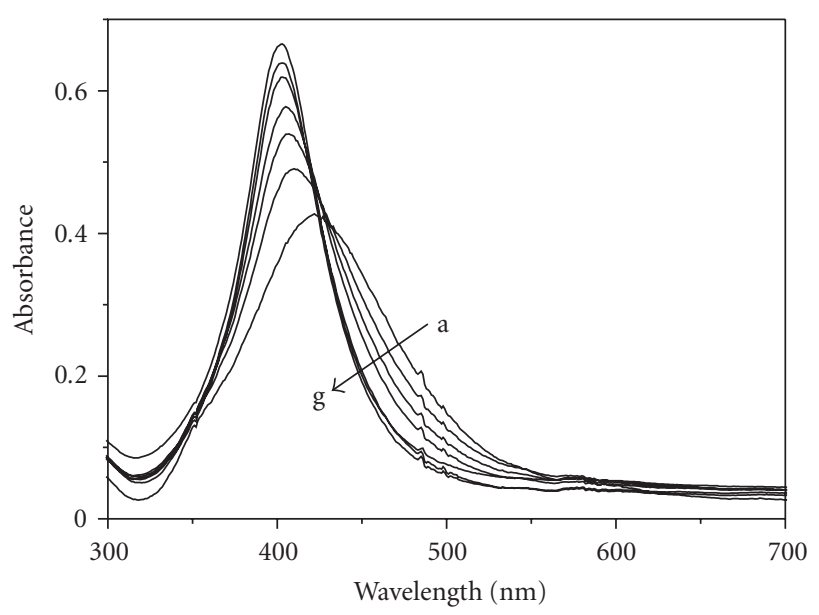

(a)

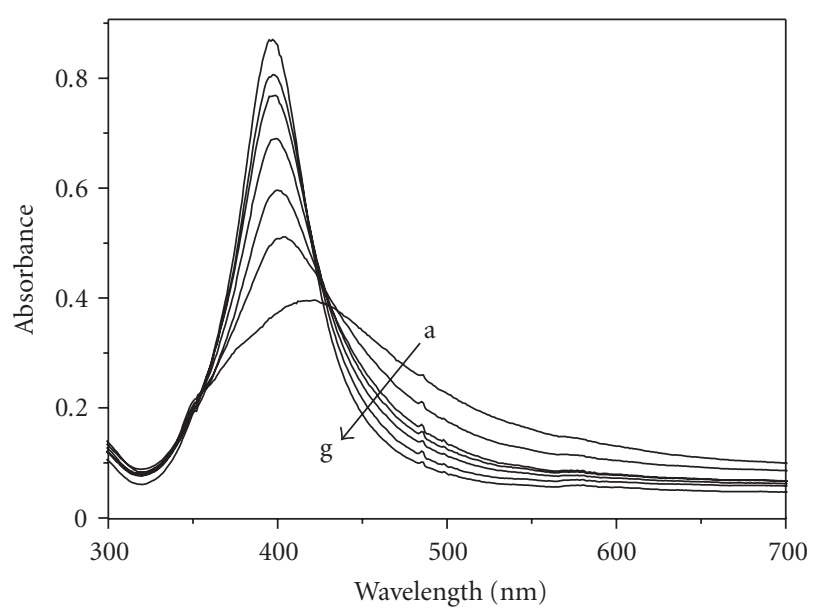

(b)

FIGURE 4: Absorption spectra of PVP and sodium citrate-protected silver nanoparticles in water as a function of the laser irradiation time; (a) PVP-stabilized samples: a $\rightarrow$ g were laser irradiated for 0, 1.0, 2.0, 4.5, 8.5, 11.5, and 14.5 minutes; (b) sodium citrate-stabilized samples: $\mathrm{a} \rightarrow$ g samples were laser irradiated for $0,1.0,2.0,3.5,5.5,7.5$, and 11.5 minutes.

Although the samples synthesized in aqueous medium did not present the same uniformity as in samples synthesized in organic solvents, the results are still very good when compared to preceding reports.

The absorption spectra of the PVP-capped silver colloids are presented in Figure 3. The surface plasmon resonance (SPR) band maximum absorption changed for the following liquid carriers: methanol and water, $398 \mathrm{~nm}$; acetone, $408 \mathrm{~nm}$; ethylene glycol, $411 \mathrm{~nm}$; and glycerin, $426 \mathrm{~nm}$. The higher the liquid dipole moment, the more the SPR band is red shifted. This result can be understood using Mie theory. The introduction of a correction to the dielectric function of the NPs that takes into account contributions from the NPs' surface and liquid media was reported elsewhere [40].

The bandwidth narrowing observed when the chemically-reduced samples are laser irradiated is presented in Figure 4 for water-based colloids, where PVP and sodium citrate were used as stabilizers. Typically, the spectra do not present detectable changes after being irradiated for 15 minutes, but all syntheses were carried out for 30 minutes. This allowed us to determine the completeness of the process.

\section{Conclusion}

Silver colloids with nearly the same average particle size $(4.3-4.9 \mathrm{~nm})$ and small dispersivity were synthesized in four organic solvents (methanol, ethylene glycol, glycerin, and acetone) using PVP as the stabilizing agent by combining chemical reduction and laser ablation. Samples prepared in water using PVP, PVA, and citrate ions as stabilizing agents also lead to good results. PVP stabilized NP's in methanol presented the narrower PSD and since the average particle size are very similar, dislocations in the maximum peak position for the SPR band is affected by the different carrier liquids.

\section{Acknowledgments}

This work was supported by INCT de Fotônica/CNPq and PROCAD/CAPES. The authors are grateful for C. A. P. Leite for TEM images and A. M. Brito-Silva acknowledges CNPq for the fellowship.

\section{References}

[1] Y. Badr and M. A. Mahmoud, "Photocatalytic degradation of methyl orange by gold silver nano-core/silica nano-shell," Journal of Physics and Chemistry of Solids, vol. 68, no. 3, pp. 413-419, 2007.

[2] C. P. Collier, R. J. Saykally, J. J. Shiang, S. E. Henrichs, and J. R. Heath, "Reversible tuning of silver quantum dot monolayers through the metal- insulator transition," Science, vol. 277, no. 5334, pp. 1978-1981, 1997.

[3] J. F. Sampaio, K. C. Beverly, and J. R. Heath, "DC transport in self-assembled 2D layers of Ag nanoparticles," Journal of Physical Chemistry B, vol. 105, no. 37, pp. 8797-8800, 2001.

[4] A. M. Whelan, M. E. Brennan, W. J. Blau, and J. M. Kelly, "Enhanced third-order optical nonlinearity of silver nanoparticles with a tunable surface plasmon resonance," Journal of Nanoscience and Nanotechnology, vol. 4, no. 1-2, pp. 66-68, 2004.

[5] K.-S. Lee and M. A. El-Sayed, "Gold and silver nanoparticles in sensing and imaging: sensitivity of plasmon response to size, shape, and metal composition," Journal of Physical Chemistry B, vol. 110, no. 39, pp. 19220-19225, 2006.

[6] G. B. Smith, C. A. Deller, P. D. Swift, A. Gentle, P. D. Garrett, and W. K. Fisher, "Nanoparticle-doped polymer foils for use in solar control glazing," Journal of Nanoparticle Research, vol. 4, no. 1-2, pp. 157-165, 2002.

[7] B.-H. Ryu, Y. Choi, H.-S. Park, et al., "Synthesis of highly concentrated silver nanosol and its application to inkjet printing," Colloids and Surfaces A, vol. 270-271, no. 1-3, pp. 345-351, 2005. 
[8] C. Damm, H. Münstedt, and A. Rösch, "The antimicrobial efficacy of polyamide 6/silver-nano- and microcomposites," Materials Chemistry and Physics, vol. 108, no. 1, pp. 61-66, 2008.

[9] G. B. Smith, C. A. Deller, P. D. Swift, A. Gentle, P. D. Garrett, and W. K. Fisher, "Nanoparticle-doped polymer foils for use in solar control glazing," Journal of Nanoparticle Research, vol. 4, no. 1-2, pp. 157-165, 2002.

[10] K. L. Kelly, E. Coronado, L. L. Zhao, and G. C. Schatz, "The optical properties of metal nanoparticles: the influence of size, shape, and dielectric environment," Journal of Physical Chemistry B, vol. 107, no. 3, pp. 668-677, 2003.

[11] M. M. Oliveira, D. Ugarte, D. Zanchet, and A. J. G. Zarbin, "Influence of synthetic parameters on the size, structure, and stability of dodecanethiol-stabilized silver nanoparticles," Journal of Colloid and Interface Science, vol. 292, no. 2, pp. 429435, 2005.

[12] Y.-S. Shon and E. Cutler, "Aqueous synthesis of alkanethiolateprotected Ag nanoparticles using bunte salts," Langmuir, vol. 20, no. 16, pp. 6626-6630, 2004.

[13] X. Z. Lin, X. Teng, and H. Yang, "Direct synthesis of narrowly dispersed silver nanoparticles using a single-source precursor," Langmuir, vol. 19, no. 24, pp. 10081-10085, 2003.

[14] X. Z. Lin, A. D. Terepka, and H. Yang, "Synthesis of silver nanoparticles in a continuous flow tubular microreactor," Nano Letters, vol. 4, no. 11, pp. 2227-2232, 2004.

[15] L. Balan, J.-P. Malval, R. Schneider, and D. Burget, "Silver nanoparticles: new synthesis, characterization and photophysical properties," Materials Chemistry and Physics, vol. 104, no. 2-3, pp. 417-421, 2007.

[16] L. C. Courrol, F. R. de Oliveira Silva, and L. Gomes, "A simple method to synthesize silver nanoparticles by photoreduction," Colloids and Surfaces A, vol. 305, no. 1-3, pp. 5457, 2007.

[17] R. He, X. Qian, J. Yin, and Z. Zhu, "Preparation of polychrome silver nanoparticles in different solvents," Journal of Materials Chemistry, vol. 12, no. 12, pp. 3783-3786, 2002.

[18] H. Yin, T. Yamamoto, Y. Wada, and S. Yanagida, "Large-scale and size-controlled synthesis of silver nanoparticles under microwave irradiation," Materials Chemistry and Physics, vol. 83, no. 1, pp. 66-70, 2004.

[19] H. S. Shin, H. J. Yang, S. B. Kim, and M. S. Lee, "Mechanism of growth of colloidal silver nanoparticles stabilized by polyvinyl pyrrolidone in $\gamma$-irradiated silver nitrate solution," Journal of Colloid and Interface Science, vol. 274, no. 1, pp. 89-94, 2004.

[20] T. Li, H. G. Park, and S.-H. Choi, " $\gamma$-irradiation-induced preparation of $\mathrm{Ag}$ and $\mathrm{Au}$ nanoparticles and their characterizations," Materials Chemistry and Physics, vol. 105, no. 2-3, pp. 325-330, 2007.

[21] R. M. Tilaki, A. Iraji-Zad, and S. M. Mahdavi, "Stability, size and optical properties of silver nanoparticles prepared by laser ablation in different carrier media," Applied Physics A, vol. 84, no. 1-2, pp. 215-219, 2006.

[22] F. Mafuné, J.-Y. Kohno, Y. Takeda, T. Kondow, and H. Sawabe, "Formation and size control of silver nanoparticles by laser ablation in aqueous solution," Journal of Physical Chemistry B, vol. 104, no. 39, pp. 9111-9117, 2000.

[23] V. Amendola, S. Polizzi, and M. Meneghetti, "Free silver nanoparticles synthesized by laser ablation in organic solvents and their easy functionalization," Langmuir, vol. 23, no. 12, pp. 6766-6770, 2007.
[24] T. Tsuji, K. Iryo, N. Watanabe, and M. Tsuji, "Preparation of silver nanoparticles by laser ablation in solution: influence of laser wavelength on particle size," Applied Surface Science, vol. 202, no. 1-2, pp. 80-85, 2002.

[25] A. Pyatenko, M. Yamaguchi, and M. Suzuki, "Synthesis of spherical silver nanoparticles with controllable sizes in aqueous solutions," Journal of Physical Chemistry C, vol. 111, no. 22, pp. 7910-7917, 2007.

[26] E. L. Falcão-Filho, C. B. de Araújo, A. Galembeck, M. M. Oliveira, and A. J. G. Zarbin, "Nonlinear susceptibility of colloids consisting of silver nanoparticles in carbon disulfide," Journal of the Optical Society of America B, vol. 22, no. 11, pp. 2444-2449, 2005.

[27] L. A. Gómez, C. B. de Araújo, A. M. Brito-Silva, and A. Galembeck, "Influence of stabilizing agents on the nonlinear susceptibility of silver nanoparticles," Journal of the Optical Society of America B, vol. 24, no. 9, pp. 2136-2140, 2007.

[28] A. M. B. Silva, C. B. de Araújo, S. Santos-Silva, and A. Galembeck, "Silver nanoparticle in situ growth within crosslinked poly(ester-co-styrene) induced by UV irradiation: aggregation control with exposure time," Journal of Physics and Chemistry of Solids, vol. 68, no. 5-6, pp. 729-733, 2007.

[29] P. V. Kamat, M. Flumiani, and G. V. Hartland, "Picosecond dynamics of silver nanoclusters. Photoejection of electrons and fragmentation," Journal of Physical Chemistry B, vol. 102, no. 17, pp. 3123-3128, 1998.

[30] W. C. Bell and M. L. Myrick, "Preparation and characterization of nanoscale silver colloids by two novel synthetic routes," Journal of Colloid and Interface Science, vol. 242, no. 2, pp. 300305, 2001.

[31] P. Šmejkal, J. Pfleger, K. Šišková, B. Vičková, O. Dammer, and M. Šlouf, "In-situ study of Ag nanoparticle hydrosol optical spectra evolution during laser ablation/fragmentation," Applied Physics A, vol. 79, no. 4-6, pp. 1307-1309, 2004.

[32] R. A. Ganeev, M. Baba, A. I. Ryasnyansky, M. Suzuki, and H. Kuroda, "Characterization of optical and nonlinear optical properties of silver nanoparticles prepared by laser ablation in various liquids," Optics Communications, vol. 240, no. 4-6, pp. 437-448, 2004.

[33] A. Henglein and M. Giersig, "Formation of colloidal silver nanoparticles: capping action of citrate arnim henglein," Journal of Physical Chemistry B, vol. 103, no. 44, pp. 95339539, 1999.

[34] S. Eustis, G. Krylova, A. Eremenko, N. Smirnova, A. W. Schill, and M. El-Sayed, "Growth and fragmentation of silver nanoparticles in their synthesis with a fs laser and CW light by photo-sensitization with benzophenone," Photochemical and Photobiological Sciences, vol. 4, no. 1, pp. 154-159, 2005.

[35] A. Takami, H. Kurita, and S. Koda, "Laser-induced size reduction of noble metal particles," Journal of Physical Chemistry B, vol. 103, no. 8, pp. 1226-1232, 1999.

[36] A. Pyatenko, M. Yamaguchi, and M. Suzuki, "Laser photolysis of silver colloid prepared by citric acid reduction method," Journal of Physical Chemistry B, vol. 109, no. 46, pp. 2160821611, 2005.

[37] H. Wang, X. Qiao, J. Chen, X. Wang, and S. Ding, "Mechanisms of PVP in the preparation of silver nanoparticles," Materials Chemistry and Physics, vol. 94, no. 2-3, pp. 449-453, 2005.

[38] Z. Zhang, B. Zhao, and L. Hu, "PVP protective mechanism of ultrafine silver powder synthesized by chemical reduction 
processes," Journal of Solid State Chemistry, vol. 121, no. 1, pp. 105-110, 1996.

[39] H. H. Huang, X. P. Ni, G. L. Loy, et al., "Photochemical formation of silver nanoparticles in poly(N-vinylpyrrolidone)," Langmuir, vol. 12, no. 4, pp. 909-912, 1996.

[40] L. A. Gómez, C. B. De Araújo, A. M. Brito-Silva, and A. Galembeck, "Solvent effects on the linear and nonlinear optical response of silver nanoparticles," Applied Physics B, vol. 92, no. 1, pp. 61-66, 2008. 

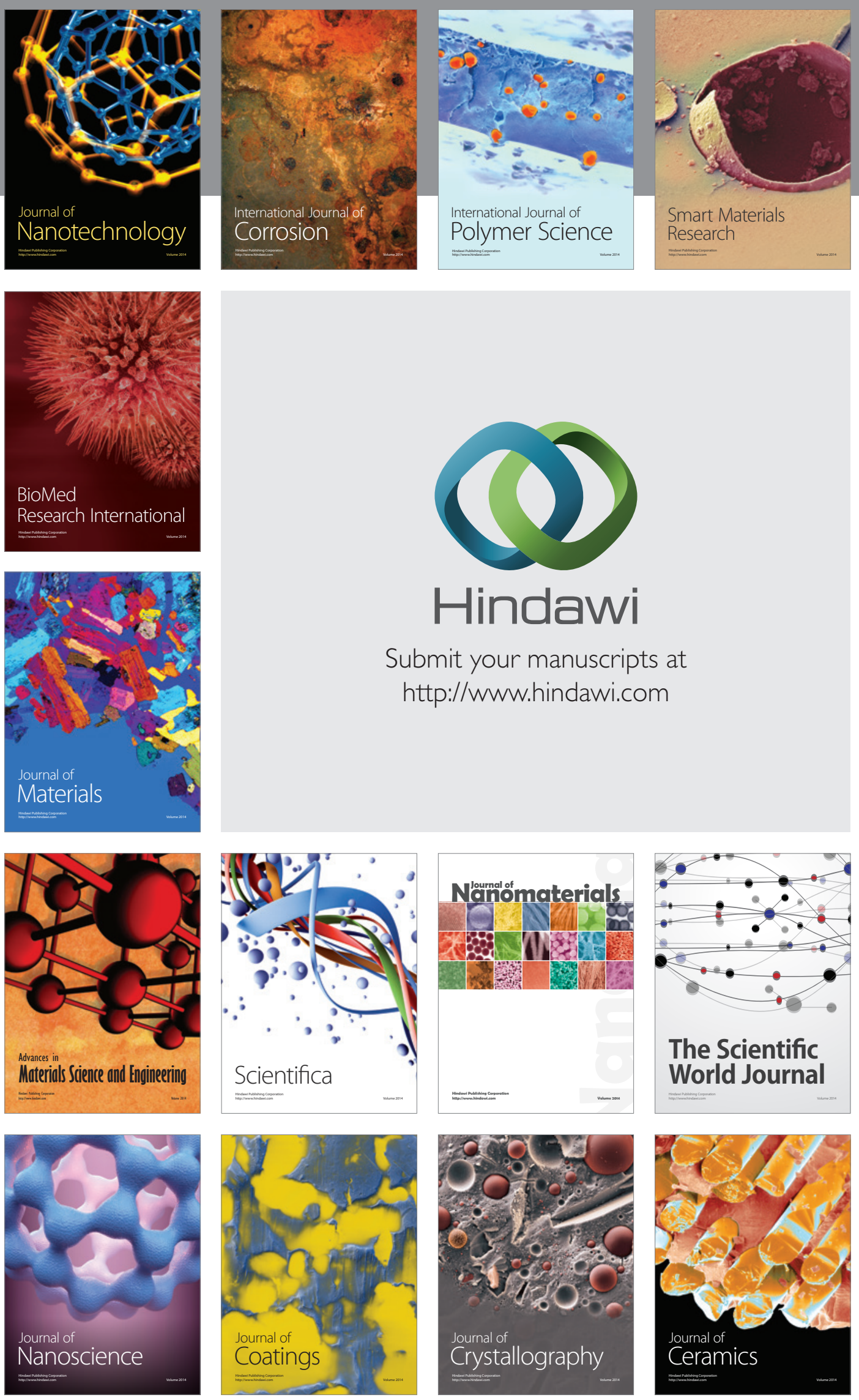

The Scientific World Journal

Submit your manuscripts at

http://www.hindawi.com

\section{World Journal}

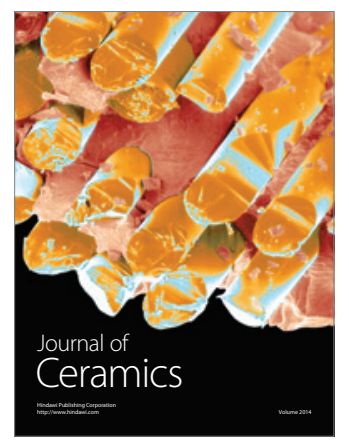

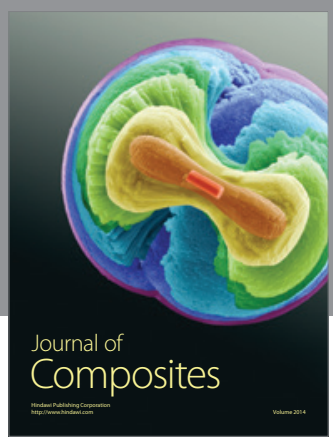
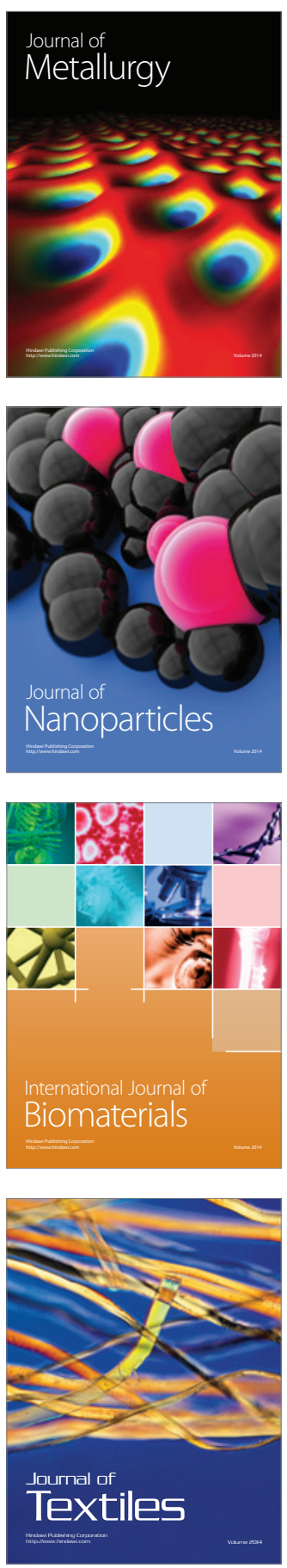